\title{
Can Energetic Terahertz Pulses Initiate Surface Catalytic Reactions on the Picosecond Time Scale?
}

\author{
Bruce D. Patterson ${ }^{\star a}$, Jacinto Sa ${ }^{\mathrm{a}}$, Anastasija Ichsanow ${ }^{\mathrm{ab}}$, Christoph P. Hauria ${ }^{\mathrm{a}}$, Carlo Vicario ${ }^{\mathrm{a}}$, \\ Clemens Rucherta, Izabela Czekaja, Regula Gehrig ${ }^{a}$, Hans C. Sigg ${ }^{a}$, Jeroen A. van Bokhoven ${ }^{\text {ab }}$, \\ Bill Pedrinia, and Rafael Abela ${ }^{a}$
}

\begin{abstract}
In preparation for picosecond pump-probe experiments at the SwissFEL X-ray laser facility, the feasibil-
\end{abstract} ity of collectively initiating surface chemical reactions using energetic pulses of terahertz radiation is being tested.

Keywords: Surface catalysis · SwissFEL $\cdot$ X-ray free electron Laser (XFEL)

\section{Introduction}

The importance of surface catalysis for modern society is immense and growing, as is evident from the demand for catalytic systems with improved efficiency, selectivity and environmental compatibility. Characteristic spatial and temporal scales in surface catalysis are dictated by the length of a chemical bond $(0.1 \mathrm{~nm})$ and by a typical molecular vibration period $(0.01$ ps). These match well the photon wavelength and pulse duration of X-ray Free Electron Lasers (XFELs), a prime example of which, the SwissFEL, ${ }^{[1]}$ is planned to go into operation at the Paul Scherrer Institute, in Würenlingen/Villigen, Switzerland, in 2017.

Pump-probe experiments at XFELs will, with the use of energy-dispersive detectors, allow single-shot measurements of near-edge X-ray absorption spectra, which will yield time-resolved information on the electronic and geometrical structure of short-lived intermediate states during a catalytic reaction. ${ }^{[2]}$ The trigger pulse for such studies should be non-ionizing, of sub-picosecond duration and capable of manipulating the local chemical environ-

\footnotetext{
${ }^{*}$ Correspondence: Prof. B. D. Patterson ${ }^{a}$ Tel.: +4156 3104524

E-mail: bruce.patterson@psi.ch

aPaul Scherrer Institut

$\mathrm{CH}-5232$ Villigen

Institute for Chemical and Bioengineering

ETH Zürich
}

ment. A candidate for such a trigger is an energetic single or few-cycle electromagnetic pulse in the terahertz $(\mathrm{THz})$ frequency region. The inclusion of a synchronized source of $\mathrm{THz}$ pump pulses is envisaged for the SwissFEL project. In preparation for these developments, we are undertaking a demonstration, on model systems, of the effectiveness of $\mathrm{THz}$ pulses to initiate surface catalytic reactions.

\section{THz and IR Initiation of Chemistry}

Various mechanisms have been discussed for the initiation of surface chemistry using THz and IR pulses. Ogasawara et al. ${ }^{[3]}$ have suggested that such initiation can proceed via a) local heating, b) resonant excitation of molecular motions or c) collective displacement of polar species. Redlich et $a .^{[4]}$ have demonstrated the IR-induced desorption of adsorbed $\mathrm{N}_{2} \mathrm{O}$ from $\mathrm{NaCl}$ (100) when an energetic pulse is resonant with an IR-active mode of the molecule. Using multiple-photon IR absorption, Hamilton et al..$^{[5]}$ have triggered a reconfiguration of $\mathrm{N}_{2} \mathrm{O}$ adsorbed on a $\mathrm{Rh}$ cluster. Experimental and theoretical studies of bio-enzymes by Masgrau et al. ${ }^{[6]}$ indicate that proton-tunneling reactions are promoted by short-range protein motions in the $\mathrm{THz}$ frequency range. Finally, avoided-level crossings induced by the Stark effect in a time-dependent electric field have been predicted by Murgida et al. ${ }^{[7]}$ to control the $\mathrm{LiNC} \leftrightarrow \mathrm{LiCN}$ isomerization reaction in the gas phase.

\section{THz-induced Dissociative Adsorption}

We consider as a model system for catalytic initiation by a $\mathrm{THz}$ pulse the dissociative adsorption of a simple molecule on a reactive surface, such as $\mathrm{CO}$ on a transition metal. At room temperature, $\mathrm{CO}$ is known ${ }^{[8]}$ to adsorb associatively, as a molecule, on the surface of transition metals to the right of $\mathrm{Co}, \mathrm{Ru}$ and $\mathrm{Re}$ in rows 4,5 and 6 of the periodic table, respectively, and to undergo dissociative adsorption on metals to the left. The adsorbed molecule is generally situated perpendicular to the surface, with its $\mathrm{C}$-atom bound atop a single metal atom. ${ }^{[9]}$ Two characteristic vibrations of the adsorbed molecule ${ }^{[10]}$ are the $\mathrm{CO}$ stretch and the hindered-translation bend modes, at frequencies of approximately 2000 and 80 $\mathrm{cm}^{-1}$, respectively. The first corresponds to an IR wavelength of $5 \mu \mathrm{m}$ and the second to a frequency of $2.5 \mathrm{THz}$.

The adsorbed $\mathrm{CO}$ has a dipole moment; on the Ni (111) surface, work function measurements ${ }^{[11]}$ yield a value of 0.28 Debye, corresponding to 0.052 elementary charges separated by the $1.13 \AA \mathrm{CO}$ bond distance. Hence, for a metal surface just to the right of the associative/dissociativeadsorption boundary, e.g. Rh, an oscillatory electric field applied parallel to the surface and which is resonant with the hindered-translation mode may excite angular excursions and $\mathrm{C}-\mathrm{O}$ bond stretching, leading to adsorptive dissociation (Fig. 1). (Note: local screening of the $\mathrm{THz}$ field by the metal surface may be avoided by using an ultrathin metallic layer on an insulating substrate.) Semi-empirical calculations for $\mathrm{CO}$ adsorbed on $\mathrm{Rh}$ (111) predict ${ }^{[12]}$ that the transition state along the absorptivedissociation reaction pathway is reached at a $70^{\circ}$ tilt and a $60 \%$ stretch. One may expect the dipole- $\mathrm{THz}$ field interaction, when resonant with the hindered-translation mode, to drive a lowering of the dissociation barrier along a direction parallel to the $\mathrm{THz}$ polarization. 


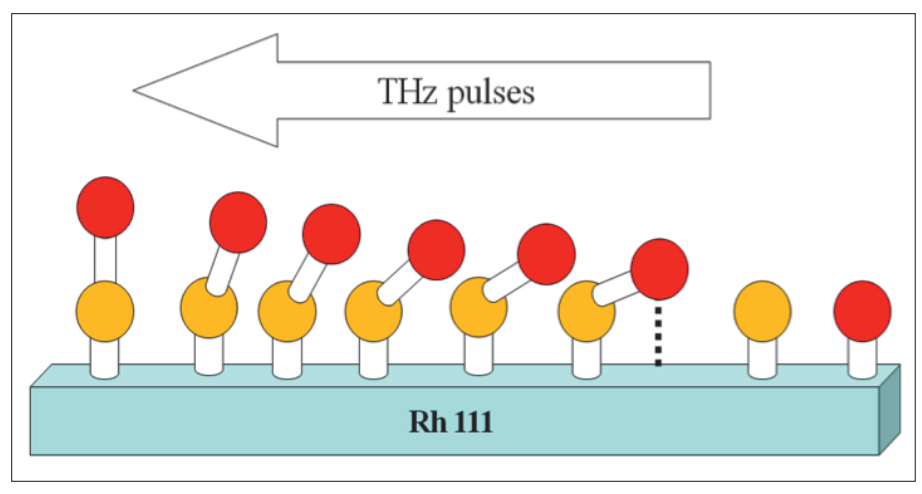

Fig. 1. A schematic representation of $\mathrm{THz}-$ induced dissociative absorption of $\mathrm{CO}$ (yellow $=\mathrm{C}$, red $=\mathrm{O}$ ) on a Rh surface.

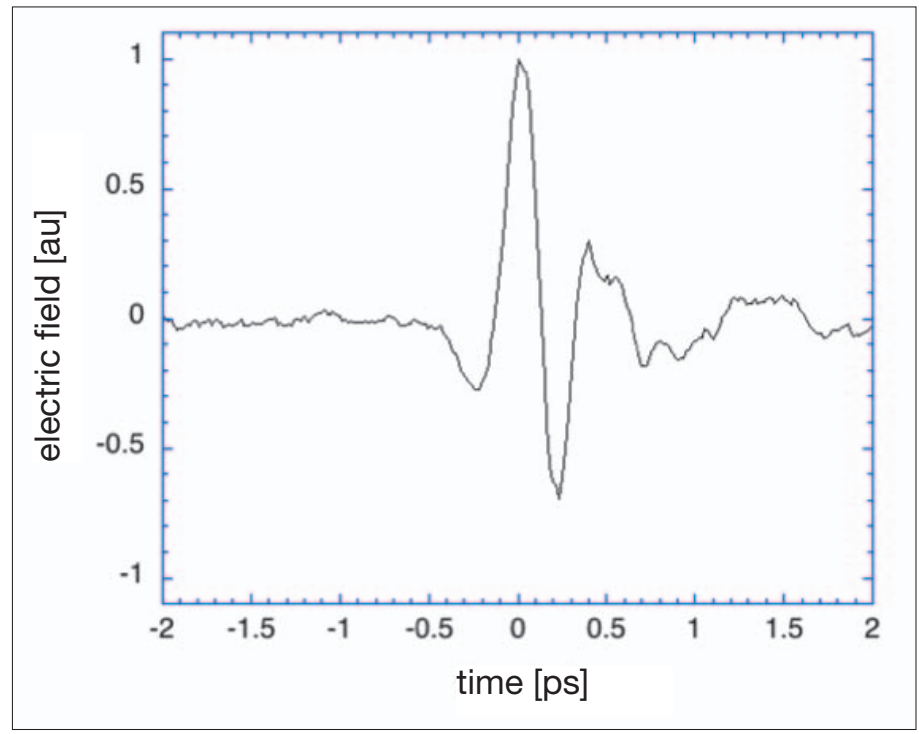

Fig. 2. An electrooptic sampling trace of the $\mathrm{THz}$ signal from the PSI optical rectification setup.
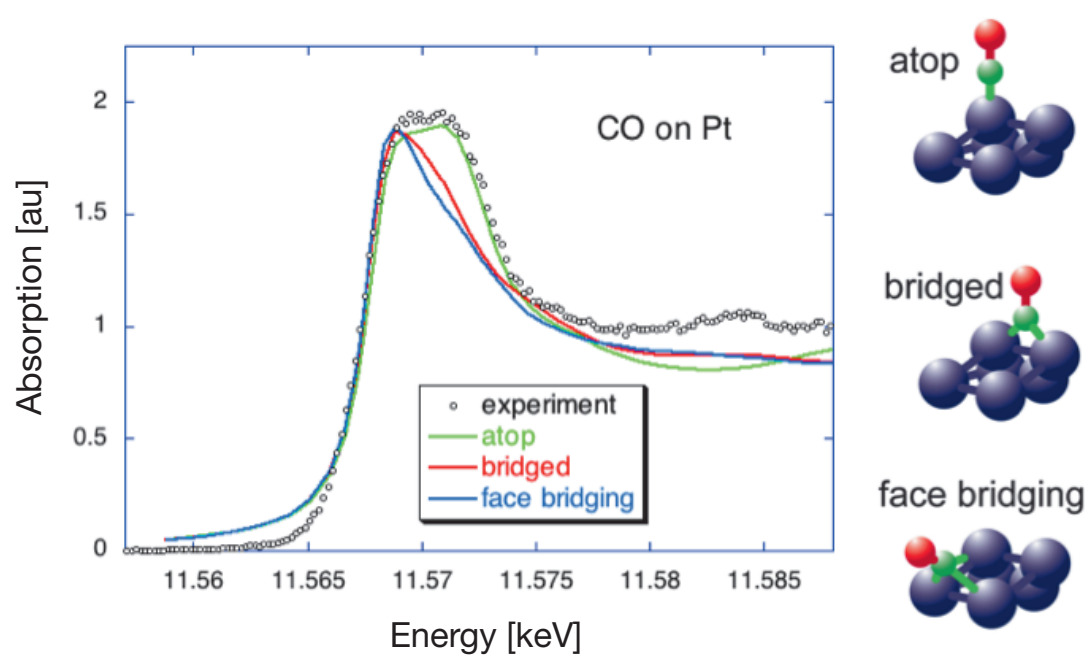

Fig. 3. A (static) near-edge X-ray absorption spectrum taken at the Pt edge at a synchrotron. ${ }^{[18]}$ From the good match with theory, it is clear that the adsorbed CO is situated in the atop position.

The influence on adsorptive dissociation of a static electric field applied perpendicular to the surface has been investigated experimentally and theoretically for the case of NO on Pt (111). Hückel calculations ${ }^{[13]}$ yield a reversed dipole moment on atop-bound $\mathrm{NO}$, i.e. with the $\mathrm{O}$ end positively charged. And with a field of $4 \mathrm{~V} / \mathrm{nm}$, electron redistribution among the molecular orbitals causes a dramatic reduction in the dissociation barrier. This is in accordance with the E-field induced dissociation observed ${ }^{[14]}$ using pulsed field desorption mass spectrometry.

In preparation for an experiment at the XFEL, we plan to observe the effect of resonant excitation of the $2.5 \mathrm{THz}$ hindered-translation mode by monitoring the frequency of the $5 \mu \mathrm{m}$ CO stretch mode in a time-resolved THz-pump/IR-probe measurement. Our preliminary DFT calculations, with non-optimized atomic positions, of adsorbed $\mathrm{CO}$ on a (111) $\mathrm{Rh}_{10}$ cluster indicate a $5 \%$ softening of the $\mathrm{CO}$ stretch frequency upon rotation of the molecular axis by $90^{\circ}$ with respect to the surface normal.

\section{Laser-based THz Generation}

Our source of energetic $\mathrm{THz}$ pulses is the laser-based optical rectification setup at the PSI-SwissFEL test injector. Incident pump pulses $(0.7 \mathrm{~mJ}, 80 \mathrm{fs})$ at 1.3 $\mu \mathrm{m}$ wavelength are produced by an optical parametric amplifier, and optical rectification to $\mathrm{THz}$ frequencies is performed in the organic crystal DAST. ${ }^{[15]}$ After focusing, the setup is presently capable of producing few cycle $\mathrm{THz}$ pulses (Fig. 2) with a peak electric field in excess of 0.3 $\mathrm{MV} / \mathrm{cm}(0.03 \mathrm{~V} / \mathrm{nm})$. Significantly stronger fields are anticipated with the use of an organic crystal with improved conversion efficiency. ${ }^{[16]}$ We are also pursuing the possibility of employing microfabricated antenna structures to dramatically increase the field strength; a 100-fold field enhancement at $1 \mathrm{THz}$ has been observed within a $70 \mathrm{~nm}$ wide slit in a $60 \mathrm{~nm}$ thick gold film. ${ }^{[17]}$

\section{THz Pump/X-ray Probe}

When the SwissFEL becomes operational, THz-pump/XFEL-probe experiments will be performed, in which a surface catalytic reaction will be initiated with a THz pulse and the subsequent time-dependent intermediate states will be probed with synchronized XFEL measurements of near-edge X-ray absorption spectroscopy ${ }^{[2,18]}$ (Fig. 3). The use of wavelength-dispersive X-ray detectors ${ }^{[19]}$ will allow the measurement of the entire near-edge absorption spectrum in a single SwissFEL shot, lasting only 20 fs. When operating in the so-called overchirped broadband mode, ${ }^{1]}$ the SwissFEL will have a relative bandwidth of $1.5 \%$, corresponding to $\pm 90 \mathrm{eV}$ at the $11.57 \mathrm{keV}$ $\mathrm{L}_{3}$-edge of platinum.

\section{Acknowledgements}

The authors wish to acknowledge ongoing discussions with A. Wokaun, M. Brown, R. Westerström and the PSI-SwissFEL team. They also acknowledge financial support from the NCCR-MUST program of the Swiss National Science Foundation.

Received: March 11, 2011

[1] B.D. Patterson, R. Abela, H.-H. Braun, U. Flechsig, R. Ganter, Y. Kim, E. Kirk, A. Oppelt, M. Pedrozzi, S. Reiche, L. Rivkin, T. Schmidt, 
B. Schmitt, V. N. Strocov, S. Tsujino, A. F Wrulich, New J. Phys. 2010, 12, 035012.

[2] B. D. Patterson, R Abela, Phys. Chem. Chem. Phys. 2010, 12, 5647.

[3] H. Ogasawara, D. Nordlund, A. Nilsson, Proc. $27^{\text {th }}$ Int. Free Electron Laser Conf., Stanford, 2005.

[4] B. Redlich, L. van der Meer, H. Zacharias, G. Meijer, G. von Helden, Nucl. Inst. Meth. Phys. Res. A 2003, 507, 556.

[5] S. M. Hamilton, W. S. Hopkins, D. J. Harding, T. R. Walsh, P. Gruene, M. Haertelt, A. Fielicke, G. Meijer, S. R. Mackenzie, J. Am. Chem. Soc. 2010, 132, 1448.

[6] L. Masgrau, A. Roujeinikova, L. O. Johannissen, P. Hothi, J. Basran, K. E. Ranaghan, A. J. Mulholland, M. J. Sutcliffe, N. S. Scrutton, D. Leys, Science 2006, 312, 237.

[7] G. E. Murgida, D.A. Wisniacki, P. I. Tamorenea, F. Borondo, Chem. Phys. Lett. 2010, 496, 356.

[8] W. Andreoni, C. M. Varma, Phys. Rev. B 1981 , 23, 437.

[9] S. E. Mason, I. Grinberg, A. M. Rappe, Phys. Rev. B 2004, 69, 161401

[10] N. V. Richardson, A. M. Bradshaw, Surf. Sci. 1979, 88,255
[11] J. C. Campuzano, R. Dus, R. G. Greenler, Surf. Sci. 1981, 102, 172.

[12] A. de Koster, R. A. van Santen, Surf. Sci. 1990 , $233,366$.

[13] H. J. Kreuzer, L. C. Wang, J. Chem. Phys. 1990, 93, 6065.

[14] N. Kruse, G. Abend, J. H. Block, J. Chem. Phys. 1988, 88, 1307

[15] A. Schneider, M. Neis, M. Stillhart, B. Ruiz, R. U. A. Khan, P. Günter, J. Opt. Soc. Am. B 2006, 23, 1822 .

[16] F. Brunner, O. Kwon, M. Jaxbinsek, A, Schneider, P. Guenter, Opt. Expr. 2008, 16, 16496.

[17] M. A. Seo, H. R. Park, S. M. Koo, D. J. Park, J. H. Kang, O. K. Suwal, S. S. Choi, P. C. M. Planken, G. S. Park, N. K. Park, Q. H. Park, D. S. Kim, Nature Photonics 2009, 3, 152.

[18] O. V. Safonova, M. Tromp, J. A. van Bokhoven, F. M. F. de Groot, J. Evans, P. Glatzel, J. Phys. Chem. B 2006, 110, 16162.

[19] D. Siddons, Z. Zhong, AIP Conf. Proc. 2004, 705,941 . 